\title{
Prevalence and antibiotic resistance of rectal Mollicutes in HIV-infected men who have sex with men at the University Hospital of Dresden, Germany
}

\author{
Petra Spornraft-Ragaller ${ }^{1}\left[\right.$ [D $\cdot$ Roger Dumke $^{2}$
}

Received: 16 August 2019 / Accepted: 20 December 2019 / Published online: 28 January 2020

(c) The Author(s) 2020

\begin{abstract}
Background Rectal sexually transmitted infections (STI) are common in men having sex with men (MSM). Mycoplasma genitalium is increasingly being reported in this localization, but due to frequent lack of symptoms at this site, clinical significance is still unclear. Rectal prevalence of Mycoplasma hominis and Ureaplasma species is not well studied so far. We aimed to investigate the prevalence and antibiotic sensitivity of rectal Mollicutes in our HIV-cohort.

Methods In 227 MSM presenting for annual STI-screening, 317 anorectal swabs were collected from January 2017 to December 2018. PCR was performed for detection of Chlamydia trachomatis, Neisseria gonorrhoeae, M. genitalium and also culture for $M$. hominis and Ureaplasma spec.

Results Prevalence for M. genitalium, M. hominis, Ureaplasma spec., C. trachomatis and N. gonorrhoeae was 8.2\%, 7.3\%, $12.0 \%, 5.1 \%$ and $1.9 \%$, respectively. Patients were asymptomatic with few exceptions. Seroprevalence of syphilis in 227 MSM was $41.9 \%$. In 20 strains of M. genitalium, resistance-associated mutations to macrolides and quinolones were found in $60 \%$ and 30\%, respectively; in five strains (25\%) to both. M. hominis and Ureaplasma spec. frequently occurred combined, mostly in significant quantity consistent with infection. M. hominis and Ureaplasma spec. regularly showed sensitivity to tetracycline. Conclusion At screening, rectal colonization with Mollicutes was common in our patients, but rarely caused symptoms. Due to rising antibiotic resistance of $M$. genitalium against quinolones, therapeutic options are increasingly limited. Treatment should be guided by antibiotic resistance testing including quinolones. In persisting anorectal symptoms, M. hominis and Ureaplasma spec. should also be taken into account.
\end{abstract}

Keywords Sexually transmitted infection $\cdot$ Ureaplasma $\cdot$ Mycoplasma $\cdot$ Resistance $\cdot$ HIV $\cdot$ MSM

\section{Introduction}

Rectal sexually transmitted infections (STI) are common in men having sex with men (MSM) and not restricted to Chlamydia trachomatis or Neisseria gonorrhoeae. Especially the cell wall-less members of the class Mollicutes have been discussed as potentially symptom- and/or transmission-relevant bacteria. The extremely slow-growing species Mycoplasma

Petra Spornraft-Ragaller

Petra.Spornraft-Ragaller@uniklinikum-dresden.de

1 Klinik und Poliklinik für Dermatologie, Universitätsklinikum Carl Gustav Carus, Technische Universität Dresden, Dresden, Germany

2 Institut für Medizinische Mikrobiologie und Hygiene, Technische Universität Dresden, Dresden, Germany genitalium is a known pathogen for nongonococcal urethritis in males and associated with cervicitis and pelvic inflammatory disease in women [1-4]. It is increasingly found in rectal swabs of MSM as well, but due to predominantly asymptomatic infections in this localization, clinical significance is still unclear. Among African women, M. genitalium is reported to facilitate HIV-infection [5, 6], but comparable studies in MSM are lacking. Treatment of $M$. genitalium infections is hampered by intrinsic resistance to betalactams, frequent occurrence of macrolide resistance and limited clinical efficiency of doxycycline. Reports on increasing quinolone resistance mainly from the Asian Pacific region currently raise concerns of spreading of multiresistant strains with strongly limited treatment options [7]. Rectal prevalence and clinical significance of Mycoplasma hominis and Ureaplasma spec. are not well studied so far. We aimed to investigate the prevalence of rectal STI and Mollicutes as 
well the antibiotic sensitivity of mycoplasma and ureaplasma species in our cohort of HIV-positive homosexual men.

\section{Methods}

In $227 \mathrm{HIV}$-infected MSM presenting for annual STIscreening, 317 anorectal swabs were collected from January 2017 to December 2018. In some patients, two swabs were available. DNA in each sample was extracted using the EZ1 automatic system (QIAGEN) and commercial realtime PCR approaches were performed for detection of $N$. gonorrhoeae, C. trachomatis, M. genitalium (TIB Molbiol) according to the recommendations of the manufacturer. In parallel, culture for propagation of M. hominis and Ureaplasma spec. was carried out. Molecular resistance testing for M. genitalium was done by partial amplification of 23S rRNA (macrolide resistance) and parC gene (quinolone resistance) and sequencing as described [8]. Mycoplasma IST test (BioMerieux) was used for cultivation and phenotypical resistance testing of $M$. hominis and Ureaplasma spec. Symptoms were evaluated at routine clinical follow up, without using a questionnaire. In all patients, seroprevalence of syphilis (TPHA $>1: 80$, VDRL $>1: 2$ ) was determined. The study was approved by the ethical committee of the TU Dresden (no 189062009).

For statistical analysis, Fisher's exact test for analysis of categorial data was performed. Because the $\mathrm{Chi}^{2}$ assumption of five or more cases in one row of the contingency table is violated for $N$. gonorrhoeae, Fischer's exact test (two-sided) was used and calculated by Stata Version 15.1.

Statistical significance is considered at a $p$ value of $\leq 0.05$.

\section{Results}

Of 317 anorectal swabs, $83(26 \%)$ were positive for at least one STI. Prevalence for M. genitalium, M. hominis, Ureaplasma spec., C. trachomatis and N. gonorrhoeae was $8.2 \%$, $7.3 \%, 12.0 \%, 5.1 \%$, and $1.9 \%$, respectively (Fig. 1). Seroprevalence of syphilis in 227 MSM (mean age 40.4 years, median age 47 years) was $41.9 \%$, but in statistical analysis this was not significantly associated with a distinctive anal coinfection. 15 patients were identified with a VDRL $>1: 2$ indicating prevalent or recent active syphilis, of whom only four had a positive rectal swab with the STI mentioned above. However, MSM with a syphilis-positive status had a $47.1 \%$ increased risk of requiring at least one additional infection compared to those with a syphilis-negative status; the highest relative risk was found for $N$. gonorrhoeae (Table 1). Although none of the associations reached statistical significance on a $5 \%$ alpha level, this is probably due to the size of the unselected sample.

Most patients with anorectal STI positive swabs were asymptomatic (85.5\%), some even in presence of $N$. gonorrhoeae and C. trachomatis. Symptoms were most frequently associated with detection of $N$. gonorrhoeae (2 of 6, 33.3\%) and $C$. trachomatis (3 of 16, 18.8\%) (Fig. 2). In 22 (26.5\%) swabs, more than one STI was detected and in four swabs (4.8\%) at least three different STI were found. Particularly M. genitalium ( $84.6 \%$ of positive swabs) and to a lesser extent Ureaplasma spec., were detected as single infection, whereas in more than half of $M$. hominis-positive swabs other STI were found as well, predominantly Ureaplasma spec. (Table 2).

Of $25 \mathrm{M}$. genitalium-positive samples, in 20 strains amplification products of targets $23 \mathrm{~S}$ rRNA and parC
Fig. 1 Prevalence of STI in 317 anorectal swabs of 227 HIVpositive patients

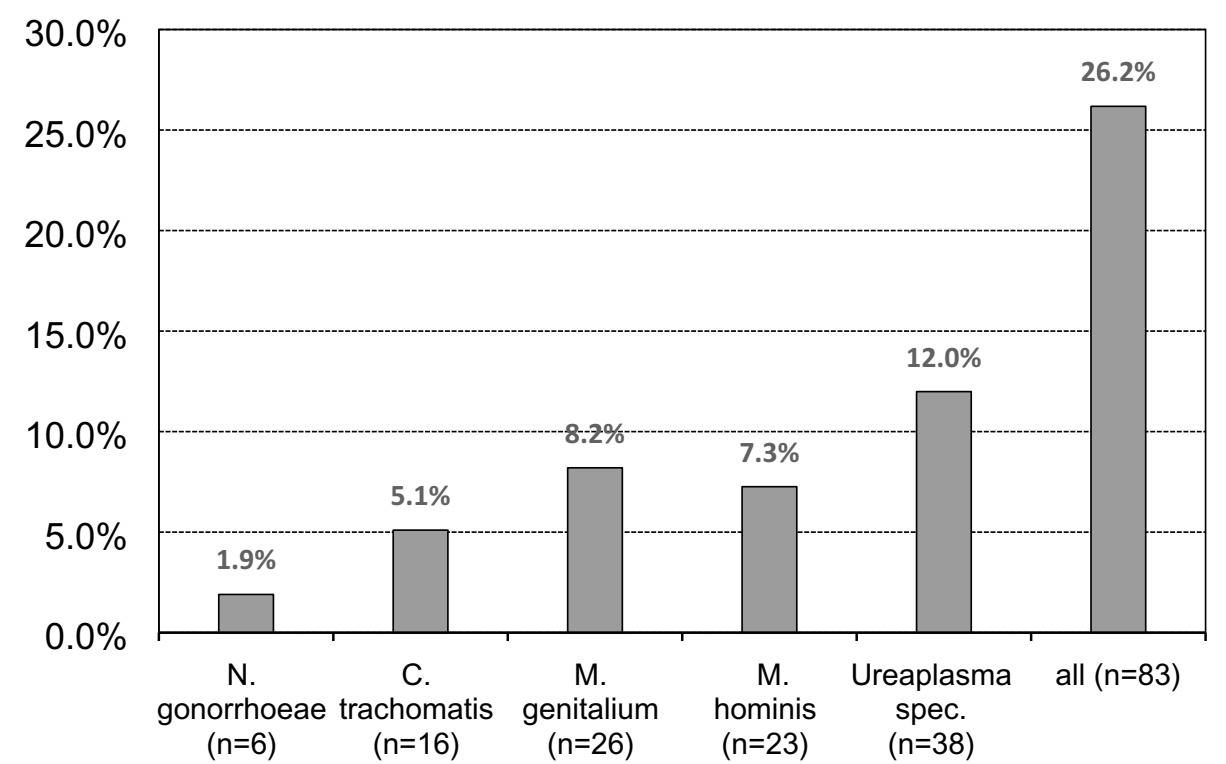


Table 1 Association of syphilisseropositive patients with detection of $N$. gonorrhoeae, $C$. trachomatis, M. genitalium, $M$. hominis, and Ureaplasma spec. in rectal swabs of HIV-positive MSM

\begin{tabular}{lrllll}
\hline Syphilis-positive $(n=95)$ & $\%$ & Syphilis-negative $(n=132)$ & $\%$ & $p$ value & RR $(95 \%$ CI $)$ \\
\hline All $(n=36)$ & 37.9 & All $(n=34)$ & 25.8 & 0.059 & $1.471(0.999-2.168)$ \\
N. gonorrhoeae $(n=5)$ & $5.3 \%$ & N. gonorrhoeae $(n=1)$ & $0.8 \%$ & 0.085 & $6.947(0.825-58.507)$ \\
C. trachomatis $(n=5)$ & $5.3 \%$ & C. trachomatis $(n=10)$ & $7.6 \%$ & 0.594 & $0.695(0.245-1.967)$ \\
M. genitalium $(n=11)$ & $11.7 \%$ & M. genitalium $(n=12)$ & $9.1 \%$ & 0.657 & $1.274(0.587-2.763)$ \\
M. hominis $(n=10)$ & $10.5 \%$ & M. hominis $(n=10)$ & $7.6 \%$ & 0.482 & $1.389(0.602-3.205)$ \\
Ureaplasma spec. $(n=19)$ & $20.0 \%$ & Ureaplasma spec. $(n=15)$ & $11.4 \%$ & 0.090 & $1.760(0.943-3.283)$
\end{tabular}

Fig. 2 Occurrence of symptoms in 83 patients tested positive for STI

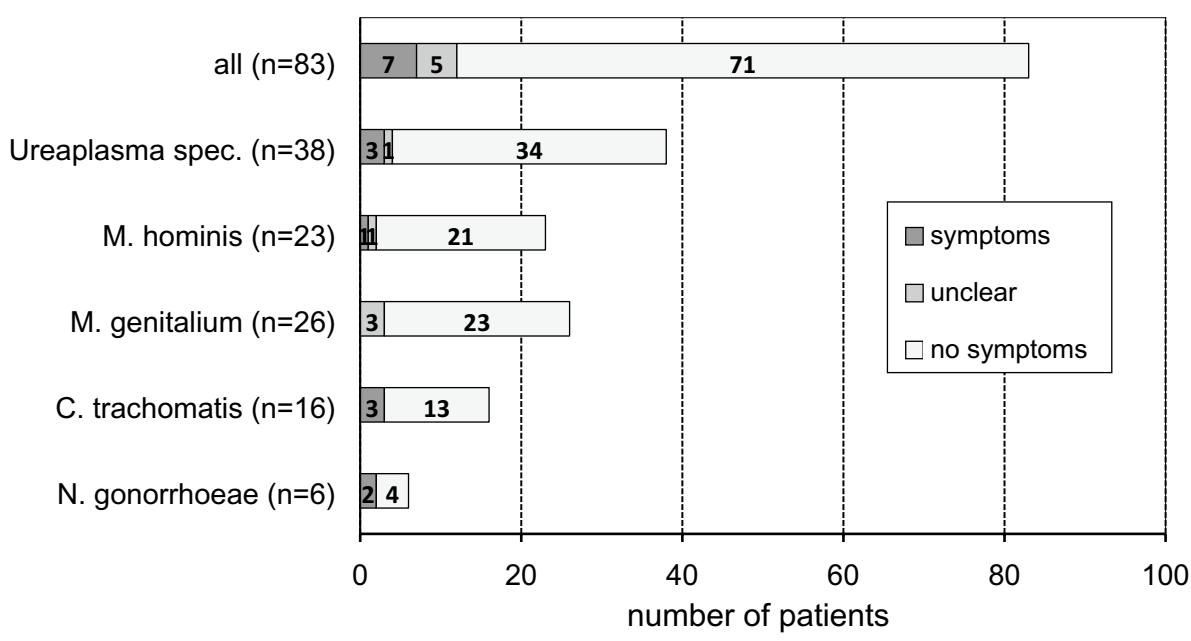

Table 2 Occurrence of Mollicutes species in 317 rectal swabs of HIV-positive MSM

\begin{tabular}{ll}
\hline Species & $\begin{array}{l}\text { Number of } \\
\text { positive sam- } \\
\text { ples }(\%)\end{array}$ \\
\hline M. genitalium & $22(6.9)$ \\
M. genitalium + M. hominis & 0 \\
M. genitalium + Ureaplasma spec. & $2(0.6)$ \\
M. genitalium + M. hominis + Ureaplasma spec. & $2(0.6)$ \\
M. genitalium + C. trachomatis & 0 \\
M. genitalium + N. gonorrhoeae & 0 \\
M. hominis & $8(2.5)$ \\
M. hominis + Ureaplasma spec. & $9(2.8)$ \\
M. hominis $+N$. gonorrhoeae & $2(0.6)$ \\
M. hominis + Ureaplasma spec. + C. trachomatis & $1(0.3)$ \\
M. hominis + Ureaplasma spec. $+C$. trachoma- & $1(0.3)$ \\
tis + N. gonorrhoeae & \\
Ureaplasma spec. & $19(6.0)$ \\
Ureaplasma spec. + C. trachomatis & $4(1.3)$ \\
Ureaplasma spec. + N. gonorrhoeae & 0 \\
\hline
\end{tabular}

could be obtained of which $60 \%$ and $30 \%$ showed resistance-associated mutations to macrolides and quinolones, respectively. Five strains (25\%) exhibited combined resistance to both macrolides and quinolones (Fig. 3). Most frequent (75\%) single-nucleotide polymorphism (SNP) in the

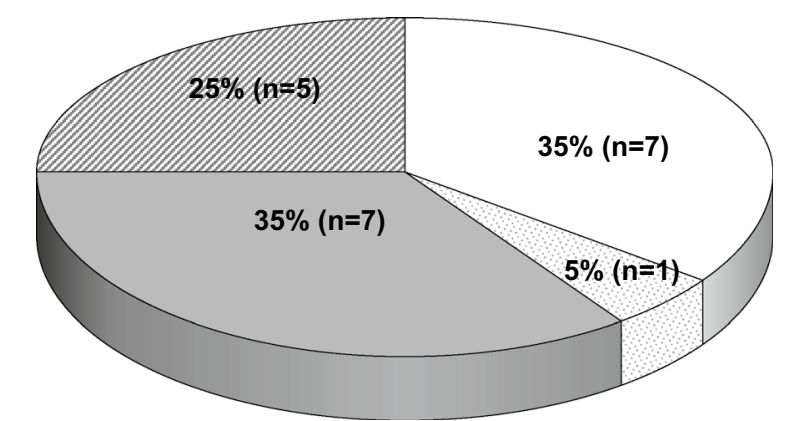

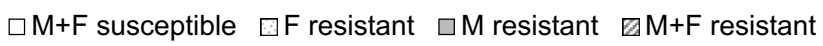

Fig. 3 Occurrence of mutations associated with macrolide (M) and

23S rRNA linked to macrolide resistance was located at position 2059 (E. coli numbering, base exchange adenine to guanine) whereas an $\mathrm{A}-\mathrm{G}$ transition at position 2058 was confirmed in the remaining three strains. Most frequent mutation in the $\operatorname{parC}$ gene associated with quinolone resistance was found at position 248 (four of six strains) leading to an amino acid change from serine to isoleucine (aa 83). Different changes were confirmed at positions 81 (glycine-cysteine) and 87 (aspartic acid to asparagine) in the quinolone-resistance determining region (QRDR) of two strains. fluorochinolone (F) resistance among $20 \mathrm{M}$. genitalium strains 
In 46 of 48 cultures, antibiotic sensitivity of $M$. hominis and Ureaplasma spec. was determined. Overall, 23 cultures of Ureaplasma spec. and 10 cultures of $M$. hominis were available. In 13 mixed cultures, material was not sufficient for differentiation between both species. Mycoplasma hominis and Ureaplasma spec. frequently occurred combined and colony forming units in culture were $>10^{4}$ in most patients, of whom some exhibited symptoms. Interestingly, in one patient complaining of anal discomfort, $U$. urealyticum (in this case, strain was typed by PCR, data not shown) was the only pathogen detected and the patient responded clinically to subsequent therapy with doxycycline. Ureaplasma spec. and $M$. hominis strains regularly showed sensitivity to tetracycline. As expected, all $M$. hominis strains were resistant against erythromycin, azithromycin, clarithromycin and most Ureaplasma spec. (20/23) against ciprofloxacin. Occurrence of tetracycline-resistant M. hominis and Ureaplasma spec. as well macrolide-resistant Ureaplasma spec. strains was not confirmed.

\section{Discussion}

In a screening situation, rectal colonization with Mollicutes was more common than with "classical" STI's in HIVinfected MSM investigated in our center. In this respect, the most frequently detected potential STI from the Mollicutes class was Ureaplasma spec. (12.0\%), followed by M. genitalium (8.2\%) and M. hominis (7.3\%), respectively. Symptoms were rare but when occurred, they seemed to be more related to Ureaplasma spec. than to M. hominis or M. genitalium. However, even confirmed $N$. gonorrhoeae and C. trachomatis infections were symptomatic in only $33 \%$ and $19 \%$ of cases, respectively. Rectal prevalence of $N$. gonorrhoeae was relatively low in our cohort, however, rectal detection of C. trachomatis (5.1\%) was equivalent to a British study screening MSM for STI (5.6\%) [9]. Seroprevalence of syphilis was comparable to the population of MSM enrolled in the German HIV seroconverter study group (39.6\%); however, these were younger (median 33 years) than our patients [10]. Due to the relatively small cohort, we not able to find a statistical significant association of syphilis seroprevalence and other rectal STI in HIV-positive MSM, but those with a syphilis-positive status had a $47.1 \%$ increased risk of acquiring at least one additional infection. Thus, syphilis may represent a proxy for increased sexual risk behaviour, leading to other STI as well.

The clinical significance of rectal Mollicutes detection is still unclear. M. genitalium is a known pathogen in nongonococcal urethritis (NGU), and suspected as involved in cervicitis and pelvic inflammatory disease [3, 11, 12]. It is most frequently found in men with symptomatic urethritis [13] with prevalence rates of $15-25 \%$ [1, 11]. In the general population, the prevalence is estimated from 1.1 to $3.9 \%[14,15]$. The microorganisms have been also detected in the anorectum of MSM where the bacteria may act as a reservoir for NGU. In a genitourinary medicine clinic in London, rectal prevalence in MSM was $4.4 \%$ and in HIVinfected MSM 14.1\% [9]. In our patients, rectal prevalence of $M$. genitalium was $8.2 \%$, which was higher than in a large German laboratory investigation on approximately 7500 patients, predominantly MSM, with a rectal prevalence of M. genitalium of 6.7\% [16] and in another German study in HIV-positive MSM attending anal cancer screening (3.3\%) [17]. Recent Australian studies reported rectal prevalence rates of M. genitalium in MSM of 7\% and 8.9\%, respectively $[2,18]$. An infection in this localization is described to be mainly asymptomatic [19], but the role of $M$. genitalium as a causative agent for proctitis is discussed controversially [2, 9, 20, 21]. In an Australian study, rectal detection of $M$. genitalium (7.0\% overall) was not more frequent in patients with symptoms of proctitis than in those without [18]. In our patients as well, no clear symptoms were associated with detection of $M$. genitalium. This is comparable with the results of a recent German study among MSM (around $50 \%$ HIV-positive), confirming anorectal symptoms in $8 \%$ of M. genitalium-positive patients [22]. Rectal and pharyngeal detection of $M$. genitalium is considered to be usually asymptomatic and thus, laboratory testing in these sites is not indicated in the general population. However, anal sampling of M. genitalium in MSM is epidemiologically important as most of infections could be missed [23] and might be transmitted if not treated.

Unfortunately, treatment of M. genitalium is increasingly complicated by the circulation of macrolide and quinoloneresistant strains worldwide. In our study, $60 \%$ and $30 \%$ of 20 strains showed mutations associated with macrolide and quinolone resistance, and 25\% with both. Prevalence of macrolide resistance of $M$. genitalium may now exceed $50 \%[3,24]$ and is reported to be even higher in HIV-positive patients [25] or HIV-PrEP users [26, 27], which corresponds to our results. The study from Germany calculated a rate of 80\% macrolide-resistant strains [22]. Quinolone resistance and treatment failures are being reported especially from the Asian Pacific region [28-30] reaching nearly $90 \%$ in China [31]. So far, data from Europe on quinolone resistance are relatively limited. Results of recent studies from England, France, Russia/Estonia, Scandinavian countries and Spain demonstrated rates of quinolone resistance of $5 \%, 6 \%, 6 \%$, $7 \%$, and $8 \%$, respectively [32-36]. Among MSM in Ireland, $33 \%$ of M. genitalium strains are quinolone-resistant [37] and in the above-mentioned German investigation, a rate of $13 \%$ was calculated [22]. Further studies have to clear, whether the different amino acid changes described in the QRDR of ParC influence the susceptibility of M. genitalium strains to quinolones. In recent reports, the common 
Ser83Ile transition was not associated with treatment failure after moxifloxacin therapy but phenotypic resistance testing resulted in significantly elevated MICs for different quinolones $[38,39]$.

In addition, in the anorectum of some of our patients, the urethral commensals Ureaplasma spec. and M. hominis could be detected by culture, mostly in significant quantity consistent with infection. Little is known on their pathogenic significance at this site and only few studies are available. In a recent Chinese study on 183 MSM, rectal prevalence of $M$. hominis detected by multiplex PCR was $7.1 \%$ [40], which is comparable with our results using culture (7.3\%). Detection rates by PCR were much higher in a study from Ireland on 107 rectal swabs from MSM: $24.3 \%$ U. urealyticum, $24.3 \%$ M. hominis, and $4.7 \%$ U. parvum, respectively [41]. Symptoms in our patients were rare and if present, mainly found in presence of Ureaplasma spec.. Of note, in one of the symptomatic patients only $U$. urealyticum was detected. This may point to a possible pathogenic significance of these bacteria under certain conditions. However, as detailed description of clinical symptoms and test of cure is missing, a clear correlation between clinical picture and detection of $U$. urealyticum in this patient cannot be established. If using the Mycoplasma IST test, determination of resistance of the isolated Ureaplasma spec. and M. hominis strains against tetracycline and macrolides provided reliable data whereas the high rate of quinolone resistance must be questioned [42]. Overall, recent European studies reported also relatively low to moderate resistance rates $(<15 \%)$ of all three species to these antibiotic classes [43, 44].

Our study has several limitations: even when our HIVcohort is still the largest in the region of Eastern Saxony, some patient selection bias can not be excluded. Anorectal symptoms were not evaluated by a questionnaire, but on clinical follow-up. Thus, some symptoms might have gone unnoticed; on the other hand, this corresponds to the normal clinical situation. Further, we did not evaluate complete antibiotic treatment, e.g., from outside of our center, so that antibiotic resistance could not be assigned to previous therapy. However, the results of the study confirm the frequent detection of members of the Mollicutes class in rectal samples of HIV-positive patients.

\section{Conclusion}

Among the sexually transmitted Mollicutes species, public health importance of $M$. genitalium is now increasingly discussed. In current European guidelines, azithromycin is recommended as first-line treatment and moxifloxacin considered being still highly effective in Europe [4]. In the meantime, this might be doubtful in an increasing number of patients. Regarding multiresistant $M$. genitalium strains, only few alternative therapy regimens with little evidence of efficacy are available. Thus, whether asymptomatic patients or partners should be treated to minimize reservoirs for transmission of M. genitalium to susceptible persons has to be discussed in the light of limited treatment options and also of recent warnings of quinolone toxicity. According to our findings, we strongly recommend resistance testing whenever possible, not only against macrolides, but against quinolones as well. To avoid induction of resistance in other species, an ongoing transmission of the pathogen and unnecessary costs, results should be available before starting therapy, especially in populations with high prevalence of $M$. genitalium such as MSM. In persisting anorectal symptoms and exclusion of well-characterized STI pathogens, M. hominis and Ureaplasma urealyticum should also be taken into account.

Acknowledgements Open Access funding provided by Projekt DEAL. We would like to thank Falko Tesch, M.Sc., Centre for Evidence-Based Healthcare, University Hospital Medizinische Fakultät Carl Gustav Carus, Technische Universität Dresden, Dresden, for reviewing our statistical analysis.

\section{Compliance with ethical standards}

Conflict of interest The authors declare that they have no conflicts of interest.

Open Access This article is licensed under a Creative Commons Attribution 4.0 International License, which permits use, sharing, adaptation, distribution and reproduction in any medium or format, as long as you give appropriate credit to the original author(s) and the source, provide a link to the Creative Commons licence, and indicate if changes were made. The images or other third party material in this article are included in the article's Creative Commons licence, unless indicated otherwise in a credit line to the material. If material is not included in the article's Creative Commons licence and your intended use is not permitted by statutory regulation or exceeds the permitted use, you will need to obtain permission directly from the copyright holder. To view a copy of this licence, visit http://creativecommons.org/licenses/by/4.0/.

\section{References}

1. Horner PJ, Martin DH. Mycoplasma genitalium infection in men. J Infect Dis. 2017;216:396-405.

2. Couldwell DL, Lewis DA. Mycoplasma genitalium infection: current treatment options, therapeutic failure, and resistanceassociated mutations. Infect Drug Resist. 2015;8:147-61.

3. Lis R, Rowhani-Rahbar A, Manhart LE. Mycoplasma genitalium infection and female reproductive tract disease: a meta-analysis. Clin Infect Dis. 2015;61:418-26.

4. Jensen JS, Cusini M, Gomberg M, et al. 2016 European guideline on Mycoplasma genitalium infections. J Eur Acad Dermatol Venereol. 2016;30:1650-6.

5. Napierala Mavedzenge S, Weiss HA. Association of Mycoplasma genitalium and HIV infection: a systematic review and meta-analysis. AIDS. 2009;23:611-20.

6. Napierala Mavedzenge S, Van Der Pol B, Weiss HA, et al. The association between Mycoplasma genitalium and HIV-1 acquisition in African women. AIDS. 2012;26:617-24. 
7. Unemo M, Jensen JS. Antimicrobial-resistant sexually transmitted infections: gonorrhoea and Mycoplasma genitalium. Nat Rev Urol. 2017;14:139-52.

8. Dumke R, Thürmer A, Jacobs E. Emergence of Mycoplasma genitalium strains showing mutations associated with macrolide and fluoroquinolone resistance in the region Dresden, Germany. Diagn Microbiol Infect Dis. 2016;86:221-3.

9. Soni S, Alexander S, Verlander N, et al. The prevalence of urethral and rectal Mycoplasma genitalium and its associations in men who have sex with men attending a genitourinary medicine clinic. Sex Transm Infect. 2010;86:21-4.

10. Jansen K, Thamm M, Bock CT, et al. High prevalence and high incidence of coinfection with Hepatitis B, Hepatitis C, and syphilis and low rate of effective vaccination against Hepatitis B in HIV-positive men who have sex with men with known date of HIV seroconversion in Germany. PLoS One. 2015;10:e0142515.

11. Taylor-Robinson D, Jensen JS. Mycoplasma genitalium: from Chrysalis to multicolored butterfly. Clin Microbiol Rev. 2011;24:498-514.

12. Manhart LE. Mycoplasma genitalium: an emergent sexually transmitted disease? Infect Dis Clin N Am. 2013;27:779-92.

13. Bradshaw CS, Tabrizi SN, Read TR, et al. Etiologies of nongonococcal urethritis: bacteria, viruses, and the association with orogenital exposure. J Infect Dis. 2006;193:336-45.

14. Sonnenberg P, Ison CA, Clifton S, et al. Epidemiology of Mycoplasma genitalium in British men and women aged 16-44 years: evidence from the third National Survey of Sexual Attitudes and Lifestyles (Natsal-3). Int J Epidemiol. 2015;44:1982-94.

15. Baumann L, Cina M, Egli-Gany D, et al. Prevalence of Mycoplasma genitalium in different population groups: systematic review and meta-analysis. Sex Transm Infect. 2018;94:255-62.

16. Blöckl N, Ruzicic S, Jessen A, et al. Mycoplasma genitalium: Prävalenz, Koinfektionen und Therapieerfolgsquote nach Erstlinienbehandlung in einem infektiologischen Zentrum in Berlin. Abstract Poster DÖAK 2019 (PW73), Abstract-Book, p. 48. http:// react-profile.org/ebook/DOEAK2019/Abstractbuch/48/. Accessed 2 Aug 2019.

17. Fuchs W, Kreuter A, Hellmich M, et al. Asymptomatic anal sexually transmitted infections in HIV-positive men attending anal cancer screening. Br J Dermatol. 2016;174:831-8.

18. Read TRH, Murray GL, Danielewski JA, et al. Symptoms, sites, and significance of Mycoplasma genitalium in men who have sex with men. Emerg Infect Dis. 2019;25:719-27.

19. Bradshaw CS, Fairley CK, Lister NA, et al. Mycoplasma genitalium in men who have sex with men at male-only saunas. Sex Transm Infect. 2009;85:432-5.

20. Bissessor M, Tabrizi SN, Bradshaw CS, et al. The contribution of Mycoplasma genitalium to the aetiology of sexually acquired infectious proctitis in men who have sex with men. Clin Microbiol Infect. 2016;22:260-5.

21. Ong JJ, Aung E, Read TRH, et al. Clinical characteristics of anorectal Mycoplasma genitalium infection and microbial cure in men who have sex with men. Sex Transm Dis. 2018;45:522-6.

22. Dumke R, Ziegler T, Abbasi-Boroudjeni N, et al. Prevalence of macrolide- and fluoroquinolone-resistant Mycoplasma genitalium strains in clinical specimens from men who have sex with men of two sexually transmitted infection practices in Berlin, Germany. Glob Antimicrob Resist. 2019;18:118-21.

23. Reinton N, Moi H, Olsen AO, et al. Anatomic distribution of Neisseria gonorrhoeae, Chlamydia trachomatis and Mycoplasma genitalium infections in men who have sex with men. Sex Health. 2013;10:199-203.

24. Getman D, Jiang A, O'Donnell M, et al. Mycoplasma genitalium prevalence, coinfection, and macrolide antibiotic resistance frequency in a multicenter clinical study cohort in the United States. J Clin Microbiol. 2016;54:2278-83.
25. Dionne-Odom J, Geisler WM, Aaron KJ, et al. High prevalence of multidrug-resistant Mycoplasma genitalium in human immunodeficiency virus-infected men who have sex with men in Alabama. Clin Infect Dis. 2018;66:796-8.

26. Couldwell DL, Jalocon D, Power M, et al. Mycoplasma genitalium: high prevalence of resistance to macrolides and frequent anorectal infection in men who have sex with men in western Sydney. Sex Transm Infect. 2018;94:406-10.

27. Deborde M, Pereyre S, Puges M, et al. High prevalence of Mycoplasma genitalium infection and macrolide resistance in patients enrolled in HIV pre-exposure prophylaxis program. Med Mal Infect. 2019;49:347-9.

28. Manhart L, Jensen JS, Bradshaw CS, et al. Efficacy of antimicrobial therapy for Mycoplasma genitalium infections. Clin Infect Dis. 2015;61:802-17.

29. Li Y, Le WJ, Li S, et al. Meta-analysis of the efficacy of moxifloxacin in treating Mycoplasma genitalium infection. Int J STD AIDS. 2017;28:1106-14.

30. Kikuchi M, Ito S, Yasuda M, et al. Remarkable increase in fluoroquinolone-resistant Mycoplasma genitalium in Japan. J Antimicrob Chemother. 2014;69:2376-82.

31. Li Y, Su X, Le W, et al. Mycoplasma genitalium in symptomatic male urethritis: macrolide use is associated with increased resistance. Clin Infect Dis. 2019. https://doi.org/10.1093/cid/ciz294.

32. Pitt R, Fifer H, Woodford N, et al. Detection of markers predictive of macrolide and fluoroquinolone resistance in Mycoplasma genitalium from patients attending sexual health services in England. Sex Transm Infect. 2018;94:9-13.

33. Le Roy C, Hénin N, Pereyre S, et al. Fluoroquinolone-resistant Mycoplasma genitalium, southwestern France. Emerg Infect Dis. 2016;22:1677-9.

34. Shipitsyna E, Rumyantseva T, Golparian D, et al. Prevalence of macrolide and fluoroquinolone resistance-mediating mutations in Mycoplasma genitalium in five cities in Russia and Estonia. PLoS One. 2017;12:e0175763.

35. Barberá MJ, Fernández-Huerta M, Jensen JS, et al. Mycoplasma genitalium macrolide and fluoroquinolone resistance: prevalence and risk factors among a 2013-2014 cohort of patients in Barcelona, Spain. Sex Transm Dis. 2017;44:457-62.

36. Unemo M, Salado-Rasmussen K, Hansen M, et al. Clinical and analytical evaluation of the new Aptima Mycoplasma genitalium assay, with data on $M$. genitalium prevalence and antimicrobial resistance in M. genitalium in Denmark, Norway and Sweden in 2016. Clin Microbiol Infect. 2018;24:533-9.

37. Mulligan V, Lynagh Y, Clarke S, et al. Prevalence, macrolide resistance and fluoroquinolone resistance in Mycoplasma genitalium in men who have sex with men (MSM) attending an STD Clinic in Dublin, Ireland in 2017-2018. Sex Transm Dis. 2019;46:e35-7.

38. Chambers LC, Jensen JS, Morgan JL, et al. Lack of association between the S83I ParC mutation in Mycoplasma genitalium and treatment outcomes among men who have sex with men with nongonococcal urethritis. Sex Transm Dis. 2019. https://doi. org/10.1097/OLQ.0000000000001035.

39. Hamasuna R, Le PT, Kutsuna S, et al. Mutations in ParC and GyrA of moxifloxacin-resistant and susceptible Mycoplasma genitalium strains. PLoS One. 2018;13:e0198355.

40. Zhao N, Li KT, Gao YY, et al. Mycoplasma genitalium and Mycoplasma hominis are prevalent and correlated with HIV risk in MSM: a cross-sectional study in Shenyang, China. BMC Infect Dis. 2019;19:494.

41. Cox C, Watt AP, McKenna JP, et al. Gardnerella vaginalis and Mollicute detection in rectal swabs from men who have sex with men. Int J STD AIDS. 2017;28:708-14. 
42. Beeton ML, Spiller OB. Antibiotic resistance among Ureaplasma spp. isolates: cause for concern? J Antimicrob Chemother. 2017;72:330-7.

43. Schneider SC, Tinguely R, Droz S, et al. Antibiotic susceptibility and sequence type distribution of Ureaplasma species isolated from genital samples in Switzerland. Antimicrob Agents Chemother. 2015;59:6026-31.

44. Meygret A, Le Roy C, Renaudin H, et al. Tetracycline and fluoroquinolone resistance in clinical Ureaplasma spp. and Mycoplasma hominis isolates in France between 2010 and 2015. J Antimicrob Chemother. 2018;73:2696-703.

Publisher's Note Springer Nature remains neutral with regard to jurisdictional claims in published maps and institutional affiliations. 